\title{
Methods for 3-D Visualization of Vascular Architecture
}

\author{
R. Dickie*, J. Lai*, M.A. Rupnick**, R. Bachoo***, H. Gonzalez*, R.A. Rogers*,**
}

*Biomedical Imaging Laboratory, Department of Environmental Health, Harvard School of Public Health, 665 Huntington Ave., Boston, MA 02115

**Vascular Biology Program, Department of Surgical Research, Children's Hospital, Karp Family Research Building, Boston, MA 02215

*** Dana Farber Cancer Institute, Mayer Bldg., 44 Binney St., Boston, MA 02115

The three dimensional architecture of blood vessel networks is of interest functionally and clinically, particularly in cancer research, but current methods for visualizing the vasculature have several limitations. Poor depth resolution and leakiness of contrast agents from the vessels can result in overestimation of the vascular volume. Traditional immunolabelling of endothelial cells has the shortcomings of depth penetration into the tissue and stability of label. Vascular corrosion casting for SEM disrupts the surrounding tissue. Here we present novel specimen preparation techniques for whole mount visualization of the 3-dimensional architecture of vessels using confocal microscopy. These methods are amenable to correlative microscopic procedures such as microCT [1], permit imaging of the vessel architecture without disruption of the surrounding tissue, and overcome some of the disadvantages associated with other vessel visualization techniques.

Our approach employed intravenous injection or cardiac perfusion of a variety of contrast agents, followed by clearing of thick sections $(>200 \mu \mathrm{m})$ of the tissues. Mice were anesthetized and heparinized, and FITC-conjugated tomato lectin (Sigma, St. Louis, MO) was administered through the tail vein or retro-orbital vein and allowed to circulate for 5 minutes. Tomato lectin is poly-Nlactosamine-specific and labels endothelial cells somewhat persistently. To achieve a more permanent label in fixed specimens, lectin administration was followed by cardiac perfusion either with a methacrylate polymer (Mercox) in which quantum dots had been suspended, or with colloidal carbon. Cardiac perfusion of saline was first performed until blanching had occurred. Animals were then perfused with 4\% paraformaldehyde, followed by $5 \mathrm{ml}$ of 1:200 solution of Maple Red semiconductor nanocrystals (Evident Technologies, Troy, NY) suspended in blue Mercox (Ladd Research Inc., Williston, VT). Mercox solution was mixed in a ratio of 3:1 mercox: diluent, using $3 \%$ cure. Mercox perfused animals were stored on ice for 20 minutes until tissue harvest. Excised tissue was fixed in 2\% paraformaldehyde, $\mathrm{pH} 7.4$ and Mercox was allowed to harden overnight in a $60^{\circ} \mathrm{C}$ water bath. Alternately, mice were perfused with Tissue Marking Dye (Fisher Scientific, Hampton, NH), a colloidal carbon.

Perfused tissue was then thick sectioned and cleared. Specimens were dehydrated through a graded methanol series, fixed in Dent's fix, bleached overnight, and cleared in Murray's clear (BABB: a solution of 1 part benzyl alcohol to 2 benzyl benzoate by volume). The BABB solution has a refractive index ( 1.55) close to that of the tissue, so tissue infiltrated with BABB becomes transparent as a uniform refractive index allows light rays to pass through with minimal scattering or absorbance. In some cases, rehydration and antibody labeling protocols using AlexaFluor secondary antibodies (Molecular Probes, Eugene, OR) were inserted into this clearing procedure after the bleaching step, using modifications of methods described in Klymkowski and Hanken [2]. Cleared, whole mount tissue was transferred to an imaging chamber filled with BABB. Specimens were examined under 10x and 20x air objectives lenses using a Leica TCS-NT confocal microscope 
fitted with argon and krypton lasers. Serial optical sections were recorded beginning at the top surface of the specimen, and the resultant stacks were rendered in three dimensions using VoxelView 2.5.1 software (Vital Images Inc., Fairfield, IA) to examine the 3-dimensional nature of the vascular network.

Dual labeling of the vasculature allows for marking of the smallest vessels and a permanent label of larger vessels. FITC lectin labels all vessels including capillaries (Figure 1A) but the signal will ultimately degrade. Carbon also labels all vessels including capillaries (Figure 2), but may interfere with routine histology of adjacent sections. Mercox fills larger vessels and, occasionally, capillaries (Figure 1B), and because the quantum dots do not lose fluorescence over time, provides long-term vascular detail. These whole mount techniques are compatible with immunostaining of other areas of interest (Figure 3).

\section{References}

[1] M. Marxen et al. Med. Phys. (2004) 31, 305.

[2] M.W. Klymkowski and J. Hanken. Methods Cell Biol. (1991) 36, 419.

[3] This research was supported by NIH PO1 CA 45548, NIH HL 65960, and NIH ES 00002.

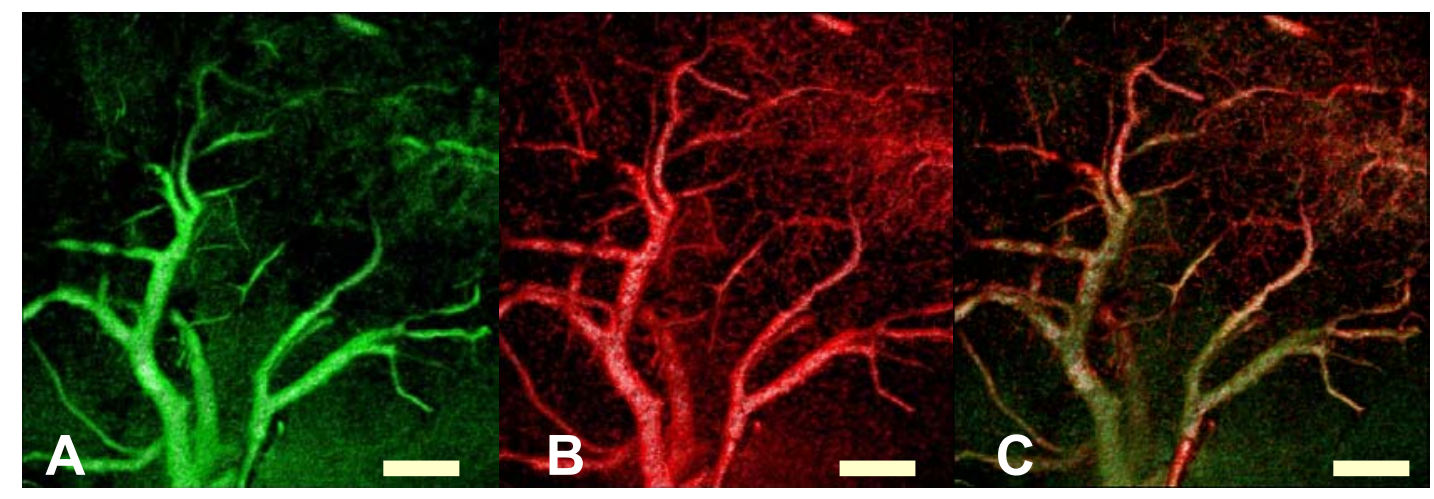

FIG. 1. Three-dimensional reconstruction from confocal stacks of brain vasculature labeled with FITC lectin marking endothelial cells (A), Mercox with quantum dots filling the vessels (B), and overlay (C). Scale bar $=100 \mu \mathrm{m}$.

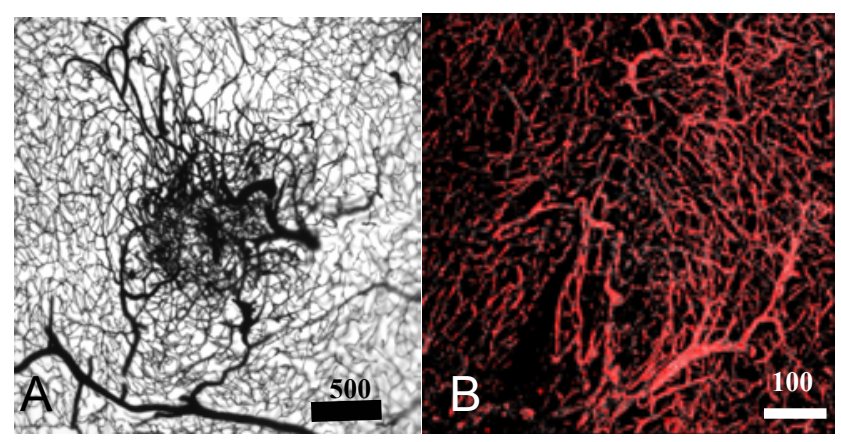

FIG. 2 A) Cleared, carbon perfused brain tumor imaged by light microscopy. B)Higher magnification 3-D rendering of carbon perfused brain tumor vasculature.

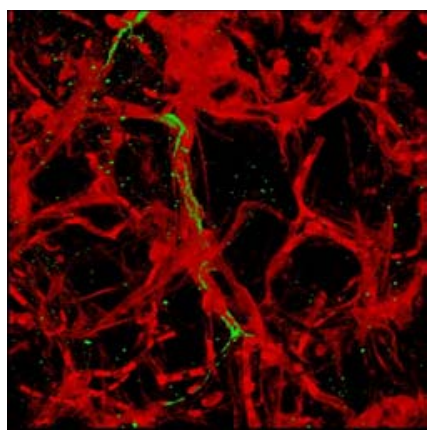

FIG. 3. $\alpha$ - smooth muscle actin antibody/Alexa (green) pericytes on endothelial cells (lectin- red) in adipose tissue 